\title{
Online Automatic and Robust Control Configuration Selection ${ }^{\star \dagger}$
}

\author{
Miguel Castaño Arranz ${ }^{1,2}$ and Wolfgang Birk ${ }^{1}$
}

\begin{abstract}
This paper presents a complete method for automatic and robust control configuration selection for linear systems which relies upon acquired process data under gaussian noise excitation.

The selection of the configuration is based on the estimation of the Interaction Measure named Participation Matrix. This estimation is derived with uncertainty bounds, which allows to determine online whether the uncertainty is sufficiently low to derive a robust decision on the control configuration to be used or if the uncertainty should be reduced by e.g. prolonging the experiment to obtain more data.
\end{abstract}

\section{INTRODUCTION}

Control Structure Selection (CSS) is normally divided in two steps: the input-output (I/O) selection and the Control Configuration Selection (CCS). In the I/O selection, the designer has to determine a set of actuators to be used by the controller to affect the system and a set of measurements to be controlled in order to track specified references. In the CCS, each actuator is associated with a set of measurements to be used by the controller for its calculation. These two steps are usually done by considering the potential of the resulting structure to achieve the proposed control goals. This paper targets the problem of CCS, for which a software tool has recently been introduced in [15]. This software targets industry application and relies upon a set of heuristic methods for CCS named Interaction Measures (IMs).

An important property of control systems in such industrial environment is robustness. The complexity of industrial processes, and the usual limitations in performing experiments at the plant are factors which are likely to increase model uncertainty. Clearly, the validity of the control configuration suggested by the IMs cannot be assessed by only analyzing the nominal model. The robustness of the CCS methods has recently received increasing attentions, like the publications on the computation of the RGA ([8], [3]), the HIIA ([11]) for uncertain process models.

The traditional methods for CCS as well as the calculation of the uncertainty bouds, require the prior access to process models with an uncertainty description. However, modeling is a time consuming task, and its complexity increases with the number of input-output channels to be modeled. The

\footnotetext{
* This work has been partially funded by: a) the Horizon 2020 OPTi project under the Grant Agreement No. 649796, b) the Horizon 2020 DISIRE project under the Grant Agreement No. 636834, c) the WARP project from the PiiA postdoc program.

$\dagger$ Authors' preprint of paper with the same title accepted for publication in the IEEE Mediterranean Conference on Control and Automation.

${ }^{1}$ Control Engineering Group, Div. of Signals and Systems, Department of Computer Science, Electrical and Space Engineering, Luleå University of Technology, SE-971 87 Luleå, Sweden

2 email: miguel.castano@1tu.se
}

aim for estimating gramian-based IMS is to removing the need of parametric process models for the analysis. In this way, The the most significant input-output channels can be determined from an experiment, and CCS can be performed. An additional advantage is that, subsequent modeling efforts can be focused on the input-output channels which are found to be significant. This would clearly avoid superfluous work, since in the traditional approach to CCS, several inputoutput channels are first modeled and later disregarded.

Despite the clear advantages, there is little previous work concerting the estimation of IMs, including the estimation of the RGA in [17] and $P M$ in [13]. The estimation results presented here include bounds on the estimation for robust CCS.

In this paper, we perform an online estimation with confidence bounds on the PM which are employed by integer programming methods to automatically design robust control configurations.

The structure of the paper is as follows. In Section II, the required preliminaries on gramian-based IMs are given. Later, in Section III, describes the estimation of PM with confidence bounds. Section IV discussed the inadequacy of the previous methods for designing control configurations with gramian-based IMs and introduces a new method for automatic CCS. In Section V the introduced automatic CCS method is extended to consider uncertainty bounds. A case study is given in Section VI, for illustrating the online estimation of PM with the application of the automatic robust method for CCS. Finally, the conclusions are given in Section VII

\section{PREliminaries ON GRAMIAN-BASEd INTERACTION MEASURES}

\section{A. Gramians for linear systems}

Assume a stable continuous-time MIMO system with $n$ inputs and $m$ outputs, represented in state space form by

$$
\begin{gathered}
\dot{x}(t)=A x(t)+B u(t) \\
y(t)=C x(t)
\end{gathered}
$$

with $A \in \Re^{q x q}, B \in \Re^{q x n}, C \in \Re^{m x q}$, where $q$ is the number of states. Alternatively, the system can be represented by the transfer function $G(s)=C(s I-A)^{-1} B$ or by the impulse response $h(t)$, which is the inverse Laplace transform of $G(s)$.

The controllability gramian $(\mathrm{P})$ and observability gramian (Q) are obtained by solving the following continuous-time Lyapunov equations (see [14]):

$$
A P+P A^{T}+B B^{T}=0
$$




$$
A^{T} Q+Q A+C^{T} C=0
$$

The eigenvalues of the controllability gramian quantify the ability to control the system states from the system inputs, and the eigenvalues of the observability gramian quantify the ability to observe the system states from the system outputs. This is reflected by the following properties (see [7]):

- The minimal energy required to transfer the states of the system from 0 to $x_{f}$ is $x_{f}^{*} P^{-1} x_{f}$.

- The maximal energy of the output obtained by observing a system with initial state $x_{0}$ is $x_{0}^{*} Q x_{0}$.

- The states which are difficult to reach are in the span of the eigenvectors of $\mathrm{P}$ which correspond to small eigenvalues, and the states which are difficult to observe are in the span of the eigenvectors of $\mathrm{Q}$ which correspond to small eigenvalues.

Consequently, gramians can be used to quantify the significance of the input-output channels and select viable control configurations which present an

\section{B. Participation Matrix}

The Participation Matrix (PM) was introduced in [12] as:

$$
P M_{i j} \triangleq \frac{\operatorname{trace}\left(P_{j} Q_{i}\right)}{\sum_{k, l=1}^{m, n} \operatorname{trace}\left(P_{k} Q_{l}\right)}=\frac{\operatorname{trace}\left(P_{j} Q_{i}\right)}{\operatorname{trace}(P Q)}
$$

where $\operatorname{trace}\left(P_{j} Q_{i}\right)$ is the trace of the product of the controllability gramian related to the jth input $P_{j}$, and the observability gramian related to the ith input $Q_{i}$ (see [12]). The sum of the elements of PM adds up to 1. Each element $P M_{i j}$ quantifies the contribution of the input-output channel $G_{i j}$ relative to the total controllability and observability of the complete system $G$.

It has been shown in [12] that, to achieve a well-decoupled system with a diagonal sensitivity function, it is necessary that the structure of the controller is the inverse of the structure of the plant. The control configuration is then designed using PM by selecting the most important inputoutput channels resulting in a model with a reduced structure which can achieve a sufficiently close to diagonal sensitivity function. The user can comprehend the amount of the total process dynamics that the reduced model is reflecting by evaluating the closeness of the sum of the selected PM values to 1 . The structure of the reduced model will be represented by a binary matrix $\Theta$.

It is important to mention that the apparent use of individual metrics on the input-output channels to evaluate the global system is possible due to the gramian decomposition. The total metric for the system is the sum of the individual metrics, and the normalization in the calculation of PM (see Eq. (2)) expresses the individual contribution as a fraction of the global contribution.

\section{Gramian-based Interaction Measures as Index Arrays}

The online estimation method discussed in Section III is particular to the PM. However, the results on automatic and robust CCS introduced in sections IV and IV are applicable to any gramian-based IM. In order to clarify this distinction, we will denote $I A$ to refer generally to any gramian-based IM.

All the gramian-based IMs are defined as an Index Array (IA) including the significance of each input-output channel divided by the total sum of the significance of all the inputoutput channels.

$$
I A_{i j}=\frac{\left[G_{i j}(s)\right]_{o p}}{\sum_{k, l=1}^{m, n}\left[G_{k l}(s)\right]_{o p}}
$$

where $[\cdot]_{o p}$ denotes the operator used by the gramian-based IM. The use of distinct operators differentiates the various gramian-based IMs. If the used operator $[\cdot]_{o p}$ is the trace of the product $P Q$, then the IA is the PM defined in Eq. (1). The Hankel Interaction Index Array (HIIA) introduced in [16] uses the Hankel Norm as operator, and $\Sigma_{2}$ introduced in [2] uses the $\mathcal{H}_{2}$-norm as operator.

\section{Online Control Configuration Selection}

A method for the estimation of $P M$ in the time domain with confidence bounds (see [6]) is now revisited. The method has been derived from previous results in [13] where a biased estimation of the $P M$ was introduced. The estimation of PM requires of a prior non-parametric estimation of the impulse response of the system which is now described.

\section{A. Impulse response estimation}

We describe now statistical properties of the estimation by linear regression of the impulse response of a linear system under gaussian noise excitation and in the presence of additive gaussian noise at the output (see [10]).

Denote by $N_{s}$ the number of logged samples of the output $y$. Collect the input $(u)$ and output histories in vectors of the form:

$$
U_{i}\left(k T_{s}\right)=\left(\begin{array}{c}
u_{1}\left(k T_{s}\right) \\
u_{1}\left(k T_{s}-T_{s}\right) \\
u_{1}\left(k T_{s}-2 T_{s}\right) \\
\vdots \\
u_{1}\left(k T_{s}-N_{i 1}^{\max } T_{s}\right) \\
u_{2}\left(k T_{s}\right) \\
u_{2}\left(k T_{s}-T_{s}\right) \\
\vdots \\
u_{n}\left(k T_{s}-N_{i n}^{\max } T_{s}\right)
\end{array}\right) ; \quad \boldsymbol{\Phi}_{i}=\left(\begin{array}{c}
U_{i}\left(T_{s}\right)^{T} \\
U_{i}\left(2 T_{s}\right)^{T} \\
\vdots \\
U_{i}\left(N_{s} T_{s}\right)^{T}
\end{array}\right)
$$

The impulse response of the output $y_{i}$ is then estimated as:

$$
\hat{\boldsymbol{H}}_{i}=\left(\boldsymbol{\Phi}_{i}^{T} \boldsymbol{\Phi}_{i}\right)^{-1} \boldsymbol{\Phi}_{i}^{T} Y_{i}
$$

where

$$
\hat{\boldsymbol{H}}_{i}=\left(\hat{h}_{i 1}(1), \ldots, \hat{h}_{i 1}\left(N_{i 1}^{\max }\right), \hat{h}_{i 2}(1), \ldots, \hat{h}_{i n}\left(N_{i n}^{\max }\right)\right)^{T}
$$

The covariance matrix of the estimated parameters is:

$$
\operatorname{Cov}\left(\hat{\boldsymbol{H}}_{i}\right)=\sigma_{y_{i}}^{2}\left(\boldsymbol{\Phi}_{i}^{T} \boldsymbol{\Phi}_{i}\right)^{-1}
$$

where $\sigma_{y_{i}}^{2}$ is the variance of the measurement noise at the output $y_{i}$. If this variance is unknown, then it can be estimated using the following unbiased statistic:

$$
\hat{\sigma}_{y_{i}}^{2}=\frac{1}{N_{s}-d_{i}}\left(Y_{i}-\boldsymbol{\Phi}_{i} \hat{\boldsymbol{H}}_{i}\right)^{2}
$$


where $d_{i}$ is the dimension of $\boldsymbol{\Phi}_{i}$.

\section{B. Estimation of $P M$ in the time domain}

Given a multivariable discrete time system, the value $\operatorname{trace}\left(P_{j} Q_{i}\right)$ can be computed as (see [13]) :

$$
\operatorname{tr}\left(P_{j} Q_{i}\right)=\sum_{k=0}^{N_{i j}} k\left(h_{i j}(k)\right)^{2}
$$

where $h_{i j}(k)$ is the true impulse response of the channel $(i, j)$ such that:

$y_{i}\left(\tau \cdot T_{s}\right)=\sum_{j=1}^{n} \sum_{k=0}^{N_{i j}} h_{i j}(k) \cdot u_{j}\left(\tau \cdot T_{s}-k \cdot T_{s}\right)$, for $\quad \tau=1,2, \ldots$

being $T_{s}$ the sampling time, and $N_{i j}$ the number of coefficients of the true impulse response of the channel $(i, j)$ until it settles to 0 .

For the estimation of $\operatorname{trace}\left(P_{j} Q_{i}\right)$ we will perform a prior estimation of the impulse response of each input-output channel using linear regression as described in Sec.III-A to match FIR filters of selected orders $N_{i j}^{\max }$ larger than the length $N_{i j}$. It will be considered for all the results in this section that the experiment for the estimation is run on a linear system and in the presence of uncorrelated Gaussian noise both at the excitation of the inputs and as additive output noise. From the estimated impulse response $\hat{h}_{i j}$, and unbiased statistic for $\operatorname{trace}\left(P_{j} Q_{i}\right)$ is (see [6]):

$$
\left.\widetilde{\operatorname{trace}\left(P_{j}\right.} Q_{i}\right)=\sum_{k=0}^{N_{i j}^{\max }} k\left(\hat{h}_{i j}(k)\right)^{2}-\sum_{k=0}^{N_{i j}^{\max }} k \cdot \sigma_{i j}^{2}(k)
$$

The variances of the estimators $\sigma_{i j}^{2}(k)$ are the diagonal elements of the covariance matrices of the linear regressions (see Eq.4).

The unbiased estimator $\left.\operatorname{trace(P_{j}} Q_{i}\right)$ is distributed as a linear combination of noncentral chi-square random variables with one degree of freedom of the form:

$$
\begin{gathered}
\left.\widetilde{\operatorname{trace}\left(P_{j}\right.} Q_{i}\right)=\sum_{k=0}^{N_{i j}^{\max }} k \cdot \sigma_{i j}^{2}(k) \cdot H_{i j}(k)-\sum_{k=0}^{N_{i j}^{\max }} k \cdot \sigma_{i j}^{2}(k) \\
H_{i j}(k) \sim \chi_{1}^{2}\left(\frac{\mu_{i j}^{2}(k)}{\sigma_{i j}^{2}(k)}\right)
\end{gathered}
$$

Under the assumption of uncorrelated output noise, the independence of the coefficients $\hat{h}_{i j}(k)$ has been ensured by using an uncorrelated excitation signal. These coefficients are independently normally distributed random variables with mean $\mu_{i j}(k)$ and variance $\sigma_{i j}^{2}(k)$. Eq. (7) is proved by introducing the random variables $\tilde{h}_{i j}(k)=\hat{h}_{i j}(k) / \sigma_{i j}(k)$ in Eq. (6).

For calculating bounds on trace $\left(P_{j} Q_{i}\right)$, the CDFs of linear combinations of independent non-central chi-square random variables have to be computed. For this purpose, the algorithm proposed in [9] can be used. For selected FIR filters significantly larger than the true impulse response, a pre-processing of the models is needed prior to the calculation of the CDFs in order to grant accurate results (see [6]).

\section{Automatic Control Configuration Selection}

\section{A. Inadequacy of previous selection methods}

The numerical matrix resulting from calculating a gramian-based IM (see Eq. (2)) has to be interpreted by the control designer in order to select an appropriate control configuration.

The following heuristic rules have been formulated in [12] for the use of the PM, and are currently applied for selecting the most significant input-output channels with the use of any gramian-based IM:

Rule 1. The simplest control configuration whose total contribution (sum of considered elements in the IA) exceeds an arbitrary threshold $\tau$ is selected as candidate. This configuration considers the input-output channels with largest significance while considering at least one inputoutput channel in each row. Control configurations designed with $\tau \leq 0.7$ are likely to derive in satisfactory performance for systems with a few sensors and actuators. As the scales of the system increase, the value of the threshold should be reduced.

Rule 2. In a hypothetical process with $r$ input-output channels where all the channels have the same contribution, this contribution will be equal to $1 / r$. This suggests that in a more heterogeneous scenario there is no benefit from considering those input-output channels for which $I A_{i j}<<1 / r$. The converse is also true, and the those input-output channels with $I A_{i j}>>1 / r$ present a significant contribution in the process dynamics.

These heuristic rules are often sufficient for the design of configurations, but they don't formulate an explicit and systematic selection method which can be implemented for an automatic selection. Furthermore, it has been observed in a CCS course given at Luleå Unviersity of Tehcnology, that the use of these heuristic rules by different designers may lead to the design of different configurations for the same system. A bottom-to-top and a top-to-bottom methods for the design of configurations are often used and are briefly explained here.

Bottom to top design of configurations. Usually, control configurations are designed by starting choosing a full decentralized configuration (one element per row and column in the IA) with the largest possible contribution and sequentially adding the channels with the largest contribution until a candidate configuration is obtained when the threshold $\tau$ is exceeded (Rule 1). This candidate configuration is often reviewed using Rule 2 by either removing insignificant channels corresponding to $I A_{i j}<<1 / r$ or adding significant channels corresponding to $I A_{i j}>>1 / r$.

Top to bottom design of configurations. An alternative was introduced in [4], where configurations are designed by starting with a full multivariable controller and inputoutput channels are sequentially removed from the reduced model in increasing order of significance until removing 
more channels would violate the threshold $\tau$. Additionally, the channels can be removed only if the resulting matrix $\theta$ representing the structure for the reduced model has full structural rank. Rule 2 is integrated in the method, by i) preventing the removal of channels with $I A_{i j}>>1 / r$ and ii) by removing channels in a last step with $I A_{i j}<<1 / r$ while preserving the structural controllability of the reduced model (full structural rank).

The following example will illustrate how both the bottomto-top and top-to-bottom approaches lead to erroneous solutions.

Example 1: Consider the Secondary Heating System introduced in [5] with transfer function:

$$
G(s)=\left(\begin{array}{ccccc}
\frac{0.0179}{s} & 0 & \frac{-0.0016}{s} & 0 & \frac{-0.0104}{s} \\
0 & \frac{-0.01}{710 s+1} & \frac{0.11}{615 s+1} & 0 & 0 \\
0 & \frac{0.00071}{s} & \frac{0.00038}{s} & \frac{-0.0012}{s} & 0 \\
0 & \frac{0.08}{1015 s+1} & \frac{-0.08}{817 s+1} & \frac{-0.36}{730 s+1} & \frac{-0.36}{604 s+1} \\
0 & \frac{-0.00047}{s} & \frac{0.00054}{s} & \frac{0.0012}{s} & \frac{0.0024}{s}
\end{array}\right)
$$

This system was presented to students in a CCS course at Luleå University of Technology where they were instructed to design a control configuration and synthesize a sparse controller. The students were introduced to Rules 1 and 2 as guidelines, and in the lack of a more explicit and systematic method they returned a variety of configurations.

We start by restricting the calculation of PM to a frequency of interest due to the existence of integrators (see [5]). Multiplying the system by a Butterworth filter of second order with bandpass $\left[10^{-3.0065} / 10,10^{-2.781} 10\right] H z$ and calculating $\mathrm{PM}$ results in:

$$
P M=\left(\begin{array}{ccccc}
0.1705 & 0 & 0.0014 & 0 & 0.0576 \\
0 & 0.0007 & 0.0954 & 0 & 0 \\
0 & 0.0844 & 0.0242 & 0.2411 & 0 \\
0 & 0.0013 & 0.0016 & 0.0343 & 0.0384 \\
0 & 0.0071 & 0.0094 & 0.0465 & 0.1861
\end{array}\right)
$$

The decentralized configuration with largest contribution is given by the pairings $u_{1}-y 1, u 3-y 2, u 4-y 3, u 4-y 2, u 5-$ $y 5$ and is represented by the following matrix $\Theta_{d e c}$ :

$$
\begin{gathered}
\Theta_{d e c}=\left(\begin{array}{ccccc}
1 & 0 & 0 & 0 & 0 \\
0 & 0 & 1 & 0 & 0 \\
0 & 0 & 0 & 1 & 0 \\
0 & 1 & 0 & 0 & 0 \\
0 & 0 & 0 & 0 & 1
\end{array}\right) \\
\left\|\theta_{d e c}\right\|_{0}=5,\left\|\Theta_{d e c} \circ P M\right\|_{1}=0.6944
\end{gathered}
$$

where $\left\|\theta_{\text {dec }}\right\|_{0}$ is the number of input-output channels in the reduced model, and $\left\|\Theta_{d e c} \circ P M\right\|_{1}$ is the sum of the considered elements of PM .

Following the bottom-to-top approach to control configuration selection, the addition of the next channel in order of significance leads to the following result for the simplest configuration with a contribution above $\tau=0.7$.

$$
\begin{gathered}
\Theta_{b t}=\left(\begin{array}{ccccc}
1 & 0 & 0 & 0 & 0 \\
0 & 0 & 1 & 0 & 0 \\
0 & 1 & 0 & 1 & 0 \\
0 & 1 & 0 & 0 & 0 \\
0 & 0 & 0 & 0 & 1
\end{array}\right) \\
\left\|\theta_{b t}\right\|_{0}=6,\left\|\Theta_{b t} \circ P M\right\|_{1}=0.778
\end{gathered}
$$

Following the top-to-bottom approach starting from a full multivariable structure results in the following simplest configuration with $\tau>0.7$ :

$$
\begin{gathered}
\Theta_{t p}=\left(\begin{array}{ccccc}
1 & 0 & 0 & 0 & 0 \\
0 & 0 & 1 & 0 & 0 \\
0 & 1 & 0 & 1 & 0 \\
0 & 0 & 0 & 0 & 1 \\
0 & 0 & 0 & 1 & 1
\end{array}\right) \\
\left\|\theta_{t p}\right\|_{0}=7,\left\|\Theta_{t p} \circ P M\right\|_{1}=0.8624
\end{gathered}
$$

The configuration $\Theta_{b t}$ includes 6 io channels, and the configuration $\Theta_{t p}$ is considering 7 io channels. Clearly, the topto-bottom approach failed in this case to find the simplest configuration with a contribution larger than $\tau=0.7$.

Consider however the following reduced model:

$$
\begin{gathered}
\Theta_{6}=\left(\begin{array}{ccccc}
1 & 0 & 0 & 0 & 0 \\
0 & 0 & 1 & 0 & 0 \\
0 & 1 & 0 & 1 & 0 \\
0 & 0 & 0 & 1 & 0 \\
0 & 0 & 0 & 0 & 1
\end{array}\right) \\
\left\|\theta_{6}\right\|_{0}=6,\left\|\Theta_{6} \circ P M\right\|_{1}=0.8118
\end{gathered}
$$

This reduced models considers 6 io channels and has a contribution of 0.8118 . Therefore, the bottom-to-top approach failed in finding the configuration with 6 channels which has the largest contribution.

This example is of large relevance, because it was previously believed that the gramian-based IMs have the Increamental property described in [1]. This means that it was accepted that the gramian-based IMs provide with a path to increase/decrease the complexity of the control configuration by adding/removing io channels in order of significance. Or in other words, it was believed that an increase in the complexity of a configuration results in a new configuration which includes all the channels of the former configuration, which is an advantage if the controller has to be reconfigured. In this example, it is shown that the best configuration with 6 io channels (see Eq. (11)) does not include all the channels of the best configuration with 5 io channels in Eq. (10). This is a counter-example which demonstrates that the gramianbased IMs are not Increamental and the methods for CCS using gramian-based IMs have to be revised.

We introduce in the sequel of this section a new automatic method for control configuration selection which overcomes with the inability of the existing bottom-to-top and top-tobottom approaches to find the adequate configuration. 
B. New method for automatic Control Configuration Selection

The configuration $\theta_{p}$ with $p$ input-output channels which can deliver the maximum contribution for a given IA can be found with the following integer program:

$$
\begin{array}{cr}
\Theta=\arg \max _{\Theta_{i j} \in\{0,1\}} & \|\Theta \circ I A\|_{1} \\
\text { subject to }: & \|\Theta\|_{0}=p \\
& \operatorname{sprank}(\Theta)=\min (m, n)
\end{array}
$$

As example, the solution of this program for the system in Eq. (8) leads to $\Theta_{d e c}$ in Eq. (10) for $p=5$ and leads to $\Theta_{6}$ in Eq. (11) for $p=6$.

However, rather than resolving the program for increasing values of $p$ until $\tau$ is exceeded, we are interested in formulating a program for finding the configuration $\Theta$ which satisfies the following:

- $\Theta$ has the minimum number of channels $p$ which can lead a contribution larger than $\tau$.

- $\Theta$ is the configuration with $p$ channels which has the largest contribution.

- $\Theta$ has full structural rank in order to guarantee structural controllability.

- $\Theta$ includes all the channels with a contribution larger than an arbitrary threshold $\Delta_{1}$.

- $\Theta$ does not include any channel with a contribution smaller than a threshold $\Delta_{2}$.

These conditions can be formulated as the integer program:

$$
\begin{array}{rr}
\Theta=\arg \max _{\Theta_{i j} \in\{0,1\}} & -\|\Theta\|_{0}+\|\Theta \circ I A\|_{1} \\
\text { subject to }: & \|\Theta \circ I A\|_{1}>\tau \\
& \operatorname{sprank}(\Theta)=\min (m, n) \\
& \text { if } I A_{i j}>\Delta_{1} \rightarrow \Theta_{i j}=1 \\
& \text { if } I A_{i j}<\Delta_{2} \rightarrow \Theta_{i j}=0
\end{array}
$$

The thresholds $\Delta_{1}$ and $\Delta_{2}$ represent the consideration of Rule 2 in the method.

This program first finds the smallest number of channels $p=\|\Theta\|_{0}$ which can derive in a contribution $\|\Theta \circ I A\|_{1}>$ $\tau$, and then finds the structure $\theta$ with $p$ channels which maximizes the contribution $\|\Theta \circ I A\|_{1}$. This is due to the fact that $p$ is an integer and $\|\Theta \circ I A\|_{1} \leq 1$.

Solving this integer program by testing all the possible configurations can lead to a burdensome computational effort, since a $5 \times 5$ system has around $29 \cdot 10^{6}$ candidate configurations. However, the number of configurations to be evaluated can dramatically be reduced by considering only the configurations which satisfy the three last inequalities in Eq. (13). More explicitly, the last two inequalities can first be used to fix values of $\Theta$, limiting the number of candidate configurations to be generated, and later we can screen the candidate configurations to preserve only those with full structural rank.

As an example, applying this program to the PM in Eq. (9) leads to the configuration in Eq. (11) for $\tau=0.7$,
$\Delta_{1}=2 /(m \cdot n)=0.08, \Delta_{2}=0.1 /(m \cdot n)=0.004$. The program can be resolved with a modern computer in a fraction of second, since generating and screening the candidate configurations as previously explained leads to only 88 candidate configurations from the original amount of approximately $29 \cdot 10^{6}$.

It is a matter of further investigations to seek additional search algorithms for systems which can potentially lead to a large number of combinations, with the use of e.g. Branch and Bound search methods.

\section{Robustification of the Automatic Control Configuration SElEction}

This section extends the automatic control configuration selection method discussed in the previous section in order to introduce the uncertainty bounds on the considered IM. This section assumes that instead of having the availability of the nominal value of an IA, we have bounds on the operator $[.]_{o p}$ used by the IA. Each value $\left[G_{i j}\right]_{o p}$ is assumed to be bounded as follows:

$$
\underline{\left[G_{i j}\right]_{o p}} \leq\left[G_{i j}\right]_{p} \leq \overline{\left[G_{i j}\right]_{o p}}
$$

We start by extending Rules 1 and 2 to the uncertain case (see [4]) resulting in the following:

Rule 3. After the selection of an arbitrary threshold $\tau$, a model which can achieve a contribution larger than $\tau$ in the worst case would be considered to reflect a robust configuration. For a given configuration $\Theta$, the minimum (worst-case) contribution is calculated as:

$$
\underline{\phi}(\Theta)=\frac{\sum_{\left\{(i, j) \mid \Theta_{i j}=1\right\}} \underline{\left[G_{i j}\right]_{o p}}}{\sum_{\left\{(i, j) \mid \Theta_{i j}=1\right\}} \underline{\left[G_{i j}\right]_{o p}}+\sum_{\left\{(i, j) \mid \Theta_{i j}=0\right\}} \overline{\left[G_{i j}\right]_{o p}}}
$$

Rule 4. The bounds on each individual element $I A_{i j}$ can be computed as:

$$
\begin{aligned}
& \underline{I A_{i j}}=\frac{\left[G_{i j}\right]_{o p}}{\underline{\left[G_{i j}\right]_{o p}}+\sum_{\substack{k=1, l=i \\
k, l) \neq(i, j)}}^{m, n} \overline{\left[G_{i j}\right]_{o p}}} \\
& \overline{I A_{i j}}=\frac{\overline{\left[G_{i j}\right]_{o p}}}{\overline{\left[G_{i j}\right]_{o p}}+\sum_{\substack{k=1, l=i \\
k, l) \neq(i, j)}}^{m, n} \underline{\left[G_{i j}\right]_{o p}}}
\end{aligned}
$$

With these bounds, robust decisions can be taken on the importance of an input-output channel. For a system with $r$ input-output channels, those input-output channels with a maximum contribution $\overline{I A_{i j}}<<r$ can be disregarded as insignificant, whilst those input-output channels with $I A_{i j}>>r$ present a significant contribution on the process $\overline{\text { dynamics. }}$

We aim to extend the nominal program in Eq. (13) to find robust configurations considering the uncertainty bounds. The goal of the program is to to he smallest subset of the input-output channels which will have a contribution larger 
than an arbitrary threshold $\tau$ when the worst-case in the uncertainty set is considered, with the following conditions:

- the controller matrix must have full structural rank,

- all channels whose best-case (maximum) contribution is larger than $\Delta_{1}$ are considered.

- no channel with a best case (larger) contribution smaller than $\Delta_{2}$ is considered.

$$
\begin{array}{r}
\Theta=\arg \max _{\Theta_{i j} \in\{0,1\}}-\|\Theta\|_{0}+\underline{\phi}(\Theta) \\
\text { subject to : } \\
\text { sprank }(\Theta)=\min (m, n) \\
\underline{\phi}(\Theta)>\tau \\
\text { if } \overline{I A_{i j}}>\Delta_{1} \rightarrow \Theta_{i j}=1 \\
\text { if } \overline{I A_{i j}}<\Delta_{2} \rightarrow \Theta_{i j}=0
\end{array}
$$

In some cases, it may be possible that the robust configuration obtained from the latter program would be simplified if the uncertainty is reduced by e.g. acquiring more process data. Denoting by $p$ as the value of $\|\theta\|_{0}$ for the obtained robust configuration in the latter program, a simpler configuration may be obtained by reducing the uncertainty if and only if the following program is feasible:

$$
\begin{array}{r}
\Theta_{p-1}=\arg \max _{\Theta_{i j} \in\{0,1\}} \bar{\phi}(\Theta) \\
\text { subject to }: \\
\operatorname{sprank}(\Theta)=m \\
\|\Theta\|_{0}=p-1 \\
\bar{\phi}(\Theta)>\tau
\end{array}
$$

where $\bar{\phi}(\Theta)$ is the best-case (maximum) contribution of the configuration $\Theta$ :

$$
\bar{\phi}(\Theta)=\frac{\sum_{\left\{(i, j) \mid \Theta_{i j}=1\right\}} \overline{\left[G_{i j}\right]_{o p}}}{\sum_{\left\{(i, j) \mid \Theta_{i j}=1\right\}} \overline{\left[G_{i j}\right]_{o p}}+\sum_{\left\{(i, j) \mid \Theta_{i j}=0\right\}} \underline{\left[G_{i j}\right]_{o p}}}
$$

If and only if there exists a solution $\Theta_{p-1}$ for this linear program, then the uncertainty set includes a simpler configuration than the robust configuration obtained from Eq. (16) and reducing the level of uncertainty might result in a simpler configuration. This feasibility test can be used to judge if a reduction of the model uncertainty (e.g. by obtaining more process data) can lead to a simplification of the robust configuration.

\section{CASE STUDY FOR THE ONLINE AUTOMATIC AND ROBUST CONTROL CONFIGURATION SELECTION}

In this section we use the method for the online estimation with confidence bounds of PM described in Sec.III and the resulting bounds are fed to the automatic and robust method for CCS introduced in Sec.V. Doing an online feasibility test of the program in Eq.17 will allow to define if we should continue acquiring process data for the possibility of simplifying the robust configuration.
Consider the process described by:

$$
G(s)=\left(\begin{array}{ccc}
\frac{2}{(s+1)(s+2)} & \frac{(2.8 s+8.4)}{(s+5)(s+2)} & \frac{-2.2}{(s+4)} \\
\frac{2}{\left(s^{2}+3 s+20\right)} & \frac{2.4}{\left(s^{2}+2 s+4\right)} & \frac{(-6 s+6)}{(s+4)(s+5)} \\
\frac{1}{(s+2)} & \frac{4}{(s+3)^{2}} & \frac{8}{(s+2)(s+5)}
\end{array}\right)
$$

The process was excited with uncorrelated Gaussian noise of variance equal to 3 , and the measurements were taken with a sampling rate of $0.1 \mathrm{sec}$ in the presence of additive uncorrelated Gaussian noise of variance 0.03 . The selected lengths for the FIR filters were 90 samples.

For a realization of the experiment, we calculate the estimation of trace $\left(P_{j} Q_{i}\right)$ as a function of the number of acquired samples, and the result is depicted in Fig. 1. The generated regions at $90 \%$ confidence are asymmetric ( $3 \%$ on the left and $7 \%$ on the right).

We apply the automatic robust CCS method described by the program in Eq. (16) with $\tau=0.7, \delta_{1}=2 / 9$ and $\delta_{2}=0.1 / 0$. The resulting configurations for different number of available samples are illustrated in Fig. 2. Each time the automatic robust CCS method is applied, the feasibility of the program in Eq.17 is tested. For a number of samples below 3932, the feasibility test is positive, indicating that acquiring more data to reduce the uncertainty might derive in a simpler configuration. For 3932 samples, the feasibility test is negative, indicating that the reduction of the model uncertainty will not lead to a simplification of the configuration and there is no need for acquiring more process data.

This example illustrates how for a low number of samples, the uncertainty is high, and there is not enough evidence to disregard most input-output channels. However, as more process data is acquired, the resulting reduced model tends to be sparser. Finally, for the designed structure using 3932 samples, the use of the $90 \%$ confidence bounds indicates that there is no simpler control configuration which can yield a total contribution larger than 0.7 . This resulting configuration corresponds to the one which would be designed for the nominal model in Eq.19 when the nominal program in Eq. (13) is applied.

\section{CONCLUSIONS}

An online automatic robust method for Control Configuration Selection has been introduced. This method requires the prior estimation with uncertainty bounds on a gramian-based IM, e.g. the Participation Matrix.

From the case study, it can be concluded that the consideration of uncertainty in the CCS problem might derive in a configuration which is more complex than the one designed for the nominal case. Large uncertainty might derive in complex robust control configurations since there is not enough evidence to discriminate any of the inputoutput channels. However, as uncertainty is reduced, the control configuration is simplified and converges to that of the nominal case. 

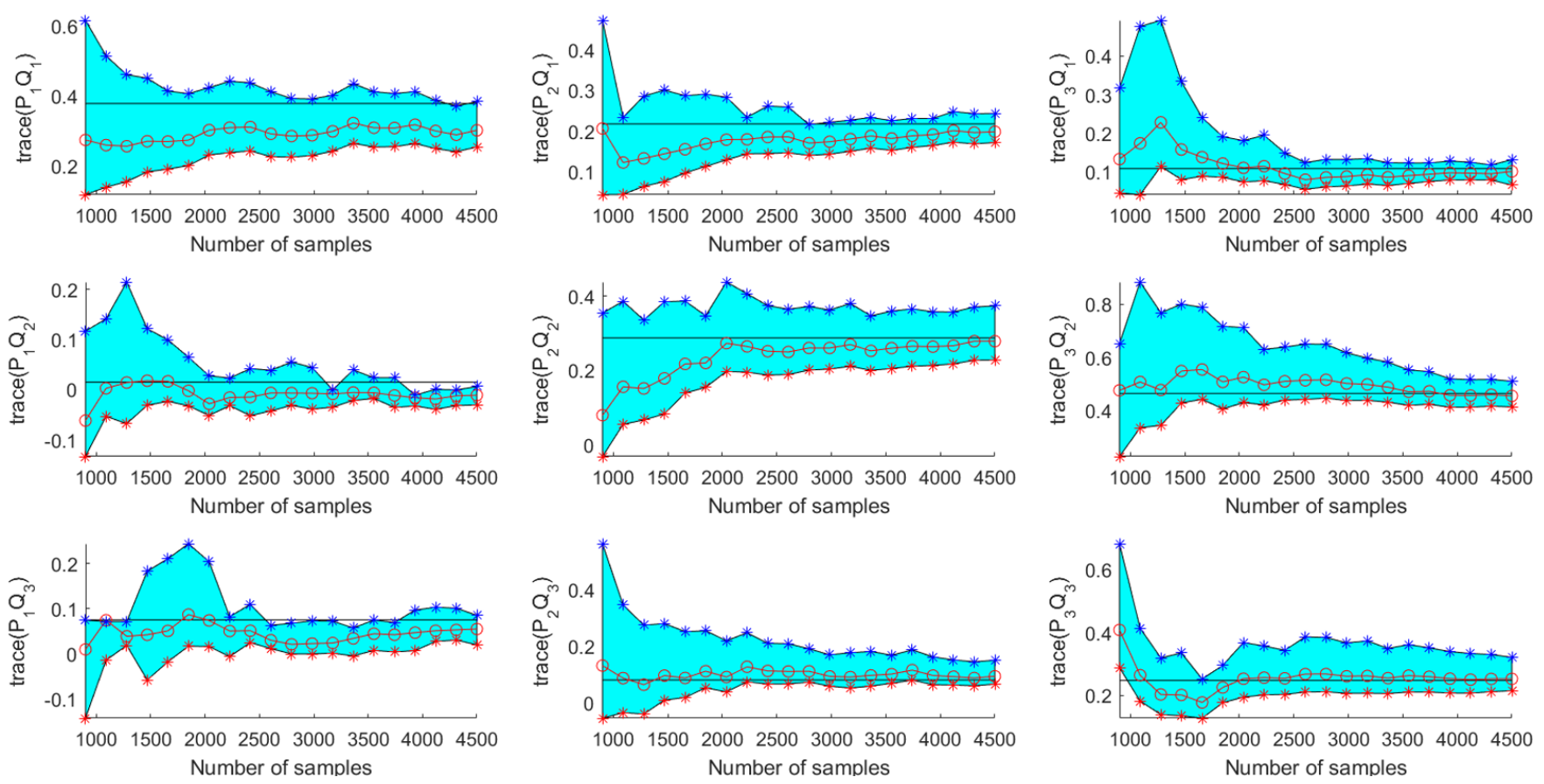

Fig. 1. Left: Estimated $\operatorname{trace}\left(P_{j} Q_{i}\right)$ for the model in Eq. (19). Estimation (circles), 90\% confidence region (shaded area) and nominal value (horizontal solid line).
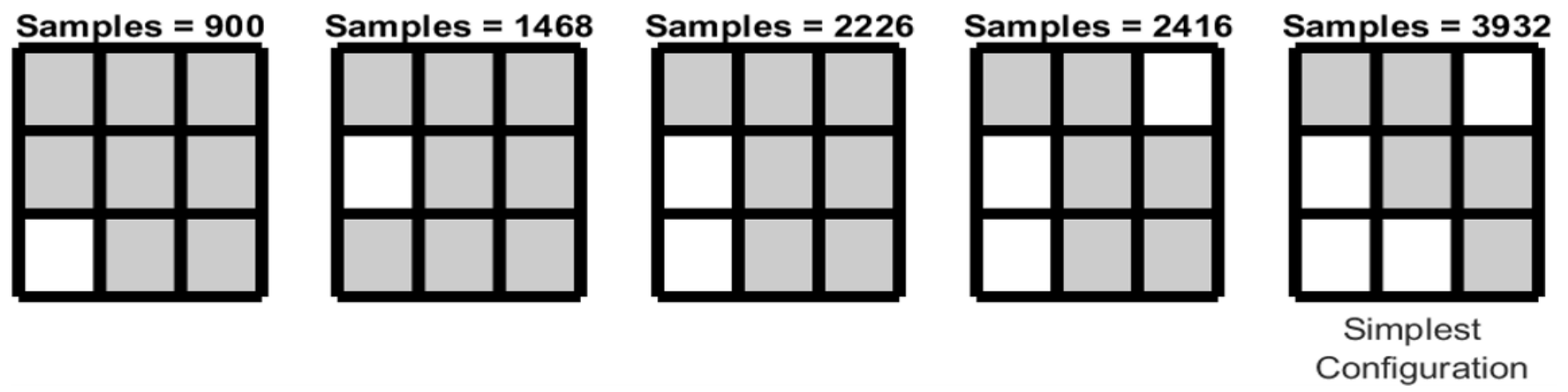

Fig. 2. Robust selection of the reduced model structure for different lengths of the acquired data.

\section{REFERENCES}

[1] Miguel Castaño Arranz. Practical tools for the configuration of control structures. PhD thesis, Luleå tekniska universitet, 2012.

[2] W. Birk and A. Medvedev. A note on gramian-based interaction measures. In Proc. of the European Control Conference 2003, University of Cambridge, UK, 2003.

[3] M. Castaño and W. Birk. A new approach to the dynamic RGA analysis of uncertain systems. In Computer-Aided Control Systems, 2008. CACSD 2008. IEEE International Conference on, pages 365370, September 2008.

[4] M. Castaño and W. Birk. On the selection of control configurations for uncertain systems using gramian-based interaction measures. Journal of Process Control, 47:213 - 225, 2016.

[5] M. Castaño, W. Birk, and P. Asplund. Control configuration selection for integrating processes using graphs. In 2015 IEEE Conference on Control Applications (CCA), pages 1606-1611, Sept 2015.

[6] M. Castaño, W. Birk, and B. Halvarsson. Empirical approach to robust gramian-based analysis of process interactions in control structure selection. In Decision and Control and European Control Conference (CDC-ECC), 2011 50th IEEE Conference on, dec. 2011.

[7] Antoulas Eolss Contribution and A. C. Antoulas. Frequency domain representation and singular value decomposition, 2002.

[8] D. Seborg D. Chen. Relative gain array analysis for uncertain process models. AIChE Journal, 48(2):302-310, 2002.
[9] R. B. Davies. The distribution of a linear combination of $\chi^{2}$ random variables, 1980.

[10] L. Ljung. System Identification. Theory for the User. Prentice Hall PTR, 2007.

[11] B. Moaveni and A. Khaki-Sedigh. Input-output pairing analysis for uncertain multivariable processes. Journal of Process Control, 18(6):527 - 532, 2008.

[12] M. E. Salgado and A. Conley. Mimo interaction measure and controller structure selection. Internation Journal of Control, 77(4):367-383, 2004.

[13] M.E. Salgado and J.I. Yuz. Mixed domain analysis of MIMO dynamic interactions. In Networking, Sensing and Control, 2007 IEEE International Conference on, pages 340-344, April 2007.

[14] Sigurd Skogestad and Ian Postlethwaite. Multivariable Feedback Control. John Wiley and Sons, second edition, 2005.

[15] W.Birk, M. Castaño, and A. Johansson. An application software for visualization and control configuration selection of interconnected processes. Control Engineering Practice, 26(0):188 - 200, 2014.

[16] Björn Wittenmark and Mario E. Salgado. Hankel-norm based interaction measure for input-output pairing. In Proc. of the 2002 IFAC World Congress, Barcelona, 2002.

[17] Chengying $\mathrm{Xu}$ and Yung C. Shin. Interaction analysis for mimo nonlinear systems based on a fuzzy basis function network model. Fuzzy Sets Syst., 158(18):2013-2025, 2007. 